\title{
Discrete symmetries in quantum electrodynamics with electric and magnetic sources
}

\author{
Elżbieta DZIMIDA-CHMIELEWSKA* \\ University of Bialystok \\ E-mail: edzimida@uwb.edu.pl \\ Jerzy A. PRZESZOWSKI \\ University of Bialystok \\ E-mail: jprzeszodalpha.uwb.edu.pl
}

QED with magnetic monopoles gives Maxwell's equations with dual symmetry and leads to the quantization of electric charge. However the transformation of parity $\mathrm{P}$ and time inversion $\mathrm{T}$ are no longer the symmetries of theory. Also the CP transformation is broken. The symmetry is restored for PT and CPT transformations. These conclusions follow from the classical Maxwell's equations and the quantum field analysis leading to the 2-point Wightman functions in Zwanziger's model of QED.

The European Physical Society Conference on High Energy Physics

18-24 July, 2013

Stockholm, Sweden

\footnotetext{
*Speaker.
} 
Quantum electrodynamics with electric and magnetic currents begins from Maxwell's equations with two kinds of external sources

$$
\vec{\nabla} \cdot \vec{E}=\frac{\rho_{e}}{\varepsilon_{0}}, \quad \vec{\nabla} \cdot \vec{B}=\mu_{0} \rho_{m}, \quad \vec{\nabla} \times \vec{E}=-\frac{\partial \vec{B}}{\partial t}-\mu_{0} \vec{K}, \quad \vec{\nabla} \times \vec{B}=\mu_{0} \varepsilon_{0} \frac{\partial \vec{E}}{\partial t}+\mu_{0} \vec{J} .
$$

Zwanziger's model of QED [1] introduces two independent gauge potentials $A_{\mu}$ and $B_{\mu}$, which describe the electromagnetic field tensor as

$$
F^{\mu v}=-n^{\mu}\left(\partial_{3} A^{v}-\partial^{v} A_{3}\right)+n^{v}\left(\partial_{3} A^{\mu}-\partial^{\mu} A_{3}\right)-\varepsilon^{\mu v 3 \rho}\left(\partial_{3} C_{\rho}-\partial_{\rho} C_{3}\right) .
$$

We make fix a space-like 4-vector $n^{\mu}=(0,0,0,1), \varepsilon^{0123}=1$ and $g_{\mu \nu}=(+,-,-,-)$. This leads to the electric and magnetic fields: $E_{i}=-\varepsilon^{0 i j 3}\left(\partial_{3} C_{j}-\partial_{j} C_{3}\right), E_{3}=\partial_{0} A_{3}-\partial_{3} A_{0}, B_{i}=-\varepsilon^{0 i j 3}\left(\partial_{3} A_{j}-\right.$ $\left.\partial_{j} A_{3}\right), B_{3}=\partial_{0} C_{3}-\partial_{3} C_{0}$, with $i, j \in\{1,2\}$. The gauge potentials $A_{\mu}$ and $C_{\mu}$ transform under gauge transformations as $A_{\mu}(x) \rightarrow A_{\mu}(x)+\partial_{\mu} \chi_{e}(x), C_{\mu}(x) \rightarrow C_{\mu}(x)+\partial_{\mu} \chi_{g}(x)$. They also transform under the electromagnetic duality: $A_{\mu} \rightarrow C_{\mu} \rightarrow-A_{\mu}$.

We want study how Maxwell's equations (1) behave under discrete transformation of the parity, the time reversal and the charge conjugation. First, the parity transformation $P$ acts on the spatial coordinates as $x=(\vec{r}, t) \stackrel{P}{\rightarrow} x^{p}=(-\vec{r}, t)$, which leads to

$$
\begin{array}{rlrl}
\rho_{a}(x) & \stackrel{P}{\longmapsto}{ }^{p} \rho_{a}(x)=\rho_{a}\left(x^{p}\right), & \vec{J}_{a}(x) & \stackrel{P}{\longmapsto}{ }^{p} \vec{J}_{a}(x)=-\vec{J}_{a}\left(x^{p}\right), \\
\vec{E}(x) & \stackrel{P}{\longmapsto}{ }^{p} \vec{E}(x)=e_{p} \vec{E}\left(x^{p}\right), & \vec{B}(x) \stackrel{P}{\longmapsto}{ }^{p} \vec{B}(x)=b_{p} \vec{B}\left(x^{p}\right),
\end{array}
$$

with $a \in\{e, m\}$. We observe that

$$
\begin{array}{lll}
\vec{\nabla} \times{ }^{p} \vec{B}(x)=\mu_{0} \varepsilon_{0} \frac{\partial}{\partial t}{ }^{p} \vec{E}(x)+\mu_{0}{ }^{p} \vec{J}_{e}(x) & \Longrightarrow & b_{p}=-e_{p}=+1 \\
\vec{\nabla} \times{ }^{p} \vec{E}(x)=-\frac{\partial}{\partial t}{ }^{p} \vec{B}(x)-\mu_{0}^{p} \vec{K}(x) & \Longrightarrow & b_{p}=-e_{p}=-1
\end{array}
$$

Therefore equations (1) do not have the parity symmetry. Similarly we proceed with the time reversal transformation $T$ which is $x=(\vec{r}, t) \stackrel{T}{\rightarrow} x^{t}=(\vec{r},-t)$. Now we have new coefficients $b_{t}$ and $e_{t}$. We find that

$$
\begin{array}{lll}
\vec{\nabla} \times{ }^{t} \vec{B}(x)=\mu_{0} \varepsilon_{0} \frac{\partial}{\partial t} t \vec{E}(x)+\mu_{0}{ }^{t} \vec{J}_{e}(x) & \Longrightarrow & b_{t}=-e_{t}=-1 \\
\vec{\nabla} \times{ }^{t} \vec{E}(x)=-\frac{\partial}{\partial t}{ }^{t} \vec{B}(x)-\mu_{0}{ }^{t} \vec{K}(x) & \Longrightarrow & b_{t}=-e_{t}=+1 .
\end{array}
$$

So we do not have time reversal symmetry either. At last we consider the charge conjugation transformation $C$, where electric and magnetic charges take their opposite values. In this case we find $b_{c}=e_{c}=-1$ for all Maxwell's equations, which means that the charge conjugation symmetry holds. We make check that $P T$ and $P C T$ transformations are the symmetries of Maxwell's equations QED with electric and magnetic sources (1) with electric end magnetic sources.

In the quantum case discrete transformations are generated by the (anti-)unitary operators: for parity we have $\mathscr{P}$, for time reversal $-\mathscr{T}$ and for charge conjugation $-\mathscr{C}$. This leads to the transformation of gauge potentials

$$
\mathscr{P} \mathscr{C} \mathscr{T} A_{\mu}(x)(\mathscr{P} \mathscr{C} \mathscr{T})^{\dagger}=-A_{\mu}(-x), \quad \mathscr{P} \mathscr{C} \mathscr{T} C_{\mu}(x)(\mathscr{P} \mathscr{C} \mathscr{T})^{\dagger}=-C_{\mu}(-x) .
$$


The vacuum state $|0\rangle$ stays invariant under $P C T$ transformation, which is described as $\mathscr{P} \mathscr{C} \mathscr{T}|0\rangle=$ $\langle 0|$, due to the anti-unitary operator $T$. This leads to the relation for the mixed Wightman function

$$
\begin{aligned}
\left\langle 0\left|A_{\mu}(x) C_{v}(0)\right| 0\right\rangle & =\left\langle 0\left|\mathscr{P} \mathscr{C} \mathscr{T} A_{\mu}(x) C_{v}(0)(\mathscr{P} \mathscr{C} \mathscr{T})^{\dagger}\right| 0\right\rangle= \\
& =\left\langle 0\left|C_{v}(x) A_{\mu}(0)\right| 0\right\rangle=-\left\langle 0\left|A_{v}(x) C_{\mu}(0)\right| 0\right\rangle,
\end{aligned}
$$

where in the last step we use the electromagnetic duality transformation. The definition (2) of $F_{\mu v}(x)$ in terms of gauge potentials $A_{\mu}$ and $C_{\mu}$, after some algebraic manipulations, gives the following relation

$$
\begin{aligned}
(n \cdot \partial)^{2} \varepsilon^{\lambda \alpha \beta \rho} \partial_{\lambda}\left\langle 0\left|A_{\alpha}(x) C_{\beta}(0)\right| 0\right\rangle & =(n \cdot \partial)\left\langle 0\left|(n \cdot F)_{\beta}(x) F^{\rho \beta}(0)\right| 0\right\rangle+ \\
& +n^{\rho}\left\langle 0\left|(n \cdot F)_{\beta}(x) \partial_{\lambda} F^{\lambda \beta}(0)\right| 0\right\rangle=2(n \cdot \partial)^{2} \partial^{\rho} D_{+}(x) .
\end{aligned}
$$

The last expression follows from the commutator function $\left\langle 0\left|\left[F_{\mu \nu}(x), F_{\lambda \rho}(0)\right]\right| 0\right\rangle$ given in [2], where we take only the positive frequency part $D_{+}$of the massless Jordan-Pauli function $D(x)$. Since $n^{2}<0$, then we can integrate out $(n \cdot \partial)^{2}$ operator and we obtain a simple equation

$$
\frac{1}{2} \varepsilon^{\mu \nu \lambda \rho} \partial_{\lambda}\left\langle 0\left|A_{\mu}(x) C_{v}(0)\right| 0\right\rangle=\partial^{\rho} D_{+}(x)
$$

We can prove that there is no Lorentz covariant solutions to this equation [3]. However we can find a special solution which is spherically symmetric.

$$
\left\langle 0\left|A_{\mu}(x) B_{v}(0)\right| 0\right\rangle_{0}=-\varepsilon_{\mu v \alpha \beta} \bar{\partial}^{\alpha} \partial^{\beta} \Delta^{-1} \star D_{+}(x) .
$$

where $\bar{\partial}^{\mu}=\partial^{\mu}-t^{\mu} \partial_{0}, t^{\mu}=(1,0,0,0)$ and

$$
\Delta^{-1}(\vec{x})=-\frac{1}{4 \pi} \frac{1}{|\vec{x}|}
$$

Then the general solution of (9) has the following form

$$
\left\langle 0\left|A_{\mu}(x) B_{v}(0)\right| 0\right\rangle=\left\langle 0\left|A_{\mu}(x) B_{v}(0)\right| 0\right\rangle_{0}+\partial_{\mu} \phi_{\nu}-\partial_{\nu} \phi_{\mu} .
$$

Here $\phi_{\mu}$ may depend on a gauge fixing condition and a fixed vector $n_{\mu}$, so this is a general form of the mixed Wightman functions for an arbitrary gauge.

We conclude that discrete symmetries lead to the strong constrains on the allowed mixed Wightman function. Though our analysis has been limited to a free field case in (8), then we may extend it also to an interacting case by means of the spectral form of the commutator functions $\left\langle 0\left|\left[F_{\mu \nu}(x), F_{\lambda \rho}(0)\right]\right| 0\right\rangle$.

\section{References}

[1] D. Zwanziger Phys.Rev. D 3880 (1971)

[2] R. E. Peierls, Proc. Roy. Soc. A 124 143(1952)

[3] E.Dzimida-Chmielewska, J. A. Przeszowski, Acta Physica Polonica B Proceedings ,Vol. 6 No. 1 p.359-364 (2012) 